ISBN: 978-1-948012-12-6

\title{
STUDY OF FATTY ACIDS IN FOOD LEGUMES WITH GAS CHROMATOGRAPHY COUPLED WITH MASS SPECTROMETRY
}

\author{
Wang Xiyue, Ming Ming, Tian Yuanyuan, Lian Lili, Zhang Hao, Lou Dawei* \\ College of Chemistry and Pharmaceutical Engineering, Jilin Institute of Chemical Technology, Chengde Street 45, Jilin, 132022, China \\ *Corresponding Author Email: dwlou@hotmail.com
}

This is an open access article distributed under the Creative Commons Attribution License, which permits unrestricted use, distribution, and reproduction in any medium, provided the original work is properly cited.

\section{ARTICLE DETAILS}

\section{Article History:}

Received 26 June 2018 Accepted 2 July 2018

Available online 1 August 2018

\begin{abstract}
In this study, a targeted method for fatty acids analysis in food legumes was developed based on gas chromatography coupled with mass spectrometry. The fatty acids were extracted with shaker in constant temperature. The effect of five extraction solvents (n-hexane, hexane/acetone (4:1), hexane/dichloromethane (4:1), hexane/isopropanol (3:2), methanol/chloroform/water (3:10:3.5)) and extraction time on efficiency of extracting fatty acids were investigated. Hexane/isopropanol (3:2) solvent and 50 min extraction time was proved to could obtain the best result. Six samples were prepared in parallel and the relative standard deviation of all fatty acids was less than $15 \%$. The linearity was good in the range of 40-140 mg. Finally the method was used to study fatty acids in corn, black bean and soybean. The result showed that the fatty acid profiling among them was significantly different. The content of most fatty acids in soybean was higher. Oleic acid and linoleic acid were the main unsaturated fatty acid. They have a higher content in corn. This method could be used to accurately quantitative analysis of fatty acid in food legumes. It provides basic data for nutritional value research and quality improvement of food legumes.
\end{abstract}

\section{KEYWORDS}

Gas chromatography coupled with mass spectrometry, fatty acid, corn, black bean, soybean.

\section{INTRODUCTION}

Corn and bean are the main oil crops, which are rich in fatty acids, such as oleic acid, linoleic acid, palmitic acid, stearic acid, etc. Fatty acids have many roles in organisms. They could provide energy for living things and play as signals transduction molecules. They also are the important part of cell membrance of organism [1]. Moreover, fatty acids play an important role in maintaining human health. Some studies found that the intake of saturated fatty acids and cholesterol in diet is closely related to the occurrence of chronic coronary heart disease, arteriosclerosis, diabetes and other diseases [2,3]. Unsaturated fatty acids such as linoleic acid and linolenic acid can reduce the risk of cardiovascular and cerebrovascular diseases, some cancers, asthma, diabetes, etc. They also have the function of enhancing immunity, improving intelligence and delaying the senescence of brain cells [4-6]. However, polyunsaturated fatty acids are prone to be oxidized to produce a fishy smell or decay, which causes the seeds to deteriorated [7]. More and more attention has been paid to the antioxidant capacity of plant seeds [8,9]. Therefore, analysis of fatty acid composition and content in plant seeds is very important to improve their nutritional value and quality [10].

The traditional fatty acid extraction is mainly carried out by Soxhlet extraction and distillation extraction to obtain crude fatty acids. Then the fatty acids are esterified by acid or alkali catalysts and detected by gas chromatography coupled with mass spectrometry [11-14]. This method requires a large number of samples and is time-consuming. Besides, the method would pollute the environment due to the use of substantial organic solvents. In this study, a pseudotargeted method based on GC-MS was developed for qualitative and quantitative analysis of fatty acids in corn, soybean and black bean. The effects of different solvents and time on the extraction of fatty acids in the samples were investigated by using the method of constant temperature oscillatory. The difference of fatty acids profile was studied by the developed method. The method was simple and required little organic solvents.

\section{MATERIALS AND METHOD}

\subsection{Solvents and instruments}

Solvents: Dichloromethane and isopropanol were purchased from Shenyang Huadong reagent factory (Shenyang, China). Methanol was obtained from Tedia company. Acetone was purchased from Tianjing Dongfang Chemical Plant. Chloroform was purchased from Tianjin North Tianyi Chemical Reagent Factory.

Instruments: Constant temperature bath oscillator was purchased from Tianjin Honour Instrument Co., Ltd (XMHS2144). XW-80A vortex was purchased from Shanghai Luxi Analytical Instruments Co., Ltd. The samples were separated with $\mathrm{H} 1650$ centrifuge (Hunan Xiangyi Experimental Instrument Development Co., Ltd). The samples were dried with nitrogen (Organomation Associates, Inc.). The sample analysis was finished with GC-MS.

\subsection{Experiment method}

Sample preparation: The corn, soybean and black bean were smashed and passed through 60 mesh sieve. $100 \mathrm{mg}$ power was weighed and put into $10 \mathrm{~mL}$ tube. $1 \mathrm{~mL}$ hexane/isopropanol (3:2, v/v) was added for extraction. Then sample was mixed by vortex for $1 \mathrm{~min}$ and oscillated at $150 \mathrm{rpm}$ for $50 \mathrm{~min}$ at room temperature. After centrifugation at $10000 \mathrm{rpm}$ for $10 \mathrm{~min}$, $700 \mu \mathrm{L}$ supernatant was dried with nitrogen. The dried sample was dissolved with $1 \mathrm{~mL}$ hexane. Then $0.25 \mathrm{~mL} \mathrm{KOH}$-methanol ( $2 \mathrm{~mol} / \mathrm{L}$ ) was added. The sample was mixed by vortex for $5 \mathrm{~min}$. The sample was neutralized by $0.25 \mathrm{~mL} \mathrm{HCl}(2 \mathrm{~mol} / \mathrm{L})$. After centrifugation at $12000 \mathrm{rpm}$ for $10 \mathrm{~min}, 700 \mu \mathrm{L}$ supernatant was obtained and dried by nitrogen. The derivatized sample was dissolved with $100 \mu \mathrm{L}$ hexane for GC-MS analysis.

GC-MS analysis: An agilent HP-5 fused silica capillary column $(30 \mathrm{~m} \times 0.25$ $\mathrm{mm} \times 0.25 \mu \mathrm{m})$ was used for sample separation. The initial oven 
temperature was $150^{\circ} \mathrm{C}$, ramped to $214^{\circ} \mathrm{C}$ at $20^{\circ} \mathrm{C} / \mathrm{min}$, then ramped to $214^{\circ} \mathrm{C}$ at $0.4^{\circ} \mathrm{C} / \mathrm{min}$, finally ramped to $280^{\circ} \mathrm{C}$ at $10^{\circ} \mathrm{C} / \mathrm{min}$ and sustained for $5 \mathrm{~min}$. The interface temperature was $260^{\circ} \mathrm{C}$. The flow rate of carrier gas (helium, 99.999\%) was $1.2 \mathrm{~mL} / \mathrm{min}$. The split ration was 10:1. $1 \mu \mathrm{L}$ sample was injected into GC-MS.

The ionization mode was electron impact at $70 \mathrm{eV}$. The temperature ionization source and transfer line were $230^{\circ} \mathrm{C}$ and $260^{\circ} \mathrm{C}$, respectively. The mass range of full scan monitoring mode was 33-600 $\mathrm{m} / \mathrm{z}$. The selective ion monitoring mode was set as following: 74, 87, 69, 83, $236 \mathrm{~m} / \mathrm{z}$ were monitored in 1-6 min; 67, 294, 296, 298, $292 \mathrm{~m} / \mathrm{z}$ were monitored in 6-8 $\mathrm{min}$; in 8-10 $\mathrm{min}, 292,74,87,326,69 \mathrm{~m} / \mathrm{z}$ were monitored; $10-19.6$ $\min , 74,87,143,382,354 \mathrm{~m} / \mathrm{z}$ were monitored.

Data processing and statistical analysis: The SIM scan table was imported to GCMS Station for metabolites quantification analysis. The two dimension peak table including retention time, quantification ion $\mathrm{m} / \mathrm{z}$ and peak intensities were obtained. Principal component analysis was carried out by MetaboAnalyst (http://www.metaboanalyst.ca/) [15]. After UV scaling, the heat-map of metabolites was got using MultiExperiment View 4.9.0.

Method validation: A certain amount of soybean, black bean and corn power was mixed to obtain QC sample. 6 samples of $100 \mathrm{mg}$ were prepared as 2.2.1. The acquired samples were performed GC-MS analysis. After data preprocessing, the relative standard deviation of fatty acids were calculated for repeatability investigation. QC sample of 40, 60, 80, 100, $120,140 \mathrm{mg}$ was weighed and prepared analyzed as 2.2.1, 2.2.2. Each sample was processed repeatedly three times. After data preprocessing, the correlation coefficient of peak area and sample amount was calculated for linearity analysis.

\section{RESULTS AND DISCUSSION}

\subsection{Evaluation of extraction solvent}

Hexane, hexane/acetone $(4: 1, v / v)$ hexane/dichloromethane $(4: 1, v / v)$, methanol/isopropanol $(3: 2, \mathrm{v} / \mathrm{v})$ and methanol/chloroform/water (3:10:3.5, v/v/v) were selected for studying the effect on extraction efficiency. $100 \mathrm{mg} \mathrm{QC}$ sample was weighed and processed as 2.2.1. The extractant was performed GC-MS analysis. The total area of saturated fatty acids and unsaturated fatty acids was used as evaluating indicator of the five extraction solvents. The result was shown in Figure 1. It could be found that the amount of saturated fatty acids and unsaturated fatty acids extracted by hexane/isopropanol was higher than other four extraction solvents. Therefore, hexane/isopropanol was chosen as extraction solvent in further experiment.
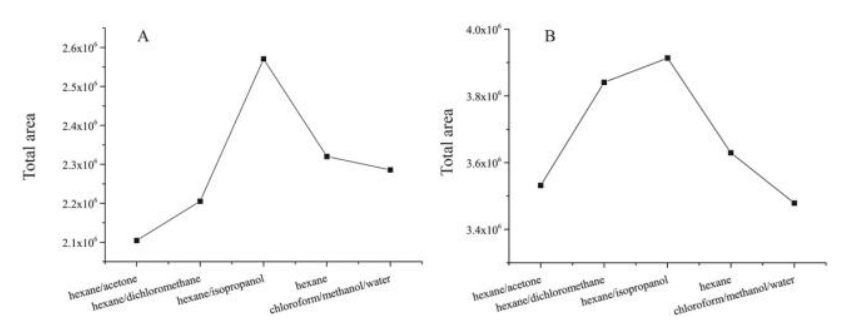

Figure 1: Comparison of total fatty acid area got by five extraction solvents. (A) saturated fatty acid, (B) unsaturated fatty acid

\subsection{The effect of extraction time}

$100 \mathrm{mg}$ QC samples were weighed and added into $1 \mathrm{~mL}$ hexane/isopropanol. They were oscillated extraction for $10 \mathrm{~min}, 20 \mathrm{~min}$, $30 \mathrm{~min}, 40 \mathrm{~min}, 50 \mathrm{~min}$ and $60 \mathrm{~min}$, respectively. The effect of shaking time on the extraction effect was investigated. The result was displayed in Figure 2. It could be seen that the content of fatty acids extracted $50 \mathrm{~min}$ is the highest, regardless of saturated fatty acids or unsaturated fatty acids. The fatty acids content decrease after $60 \mathrm{~min}$ of oscillatory extraction, which may be caused by the volatilization of extraction solvent for a long time.
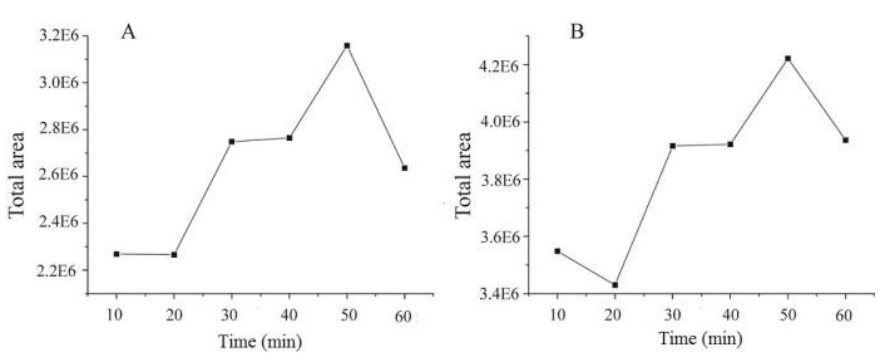

Figure 2: Investigation of extraction time. (A) saturated fatty acid, (B) unsaturated fatty acid

\subsection{Validation of the method}

Six samples were processed in parallel as 2.2.1 and analyzed by GC-MS. The peak area of each fatty acid was obtained after data pretreatment. The relative standard deviation was calculated and the repeatability of the method was investigated. The results showed that the relative standard deviations of all fatty acids were less than $15 \%$, which indicated that the method had good repeatability. The linear correlation coefficients between the peak area of fatty acids and their corresponding samples amount were all greater than 0.9. It indicated that there was a good linear relationship between the response of fatty acids and the amount of samples in the range of $40-140 \mathrm{mg}$. The results of repeatability and linearity showed that the method has good stability and reliability and it could be used for accurately quantitative analysis of fatty acids.

\subsection{The application of the method}

The developed method was used for analysis of fatty acids in corn, soybean and black bean. The obtained peak area of fatty acid was normalized by total area and the difference of fatty acid profile was further analyzed by multivariate statistics method. The score plot of the principal component analysis (PCA) of fatty acid profile in corn, soybean and black bean was shown in Figure 3. It could be seen that there was distinct separation between corn and soybean, black bean in the PC1 direction. It indicated that the big difference exists among the samples. The soybean and black bean had a little separation in the PC2 direction, which indicated that there was small difference between them.

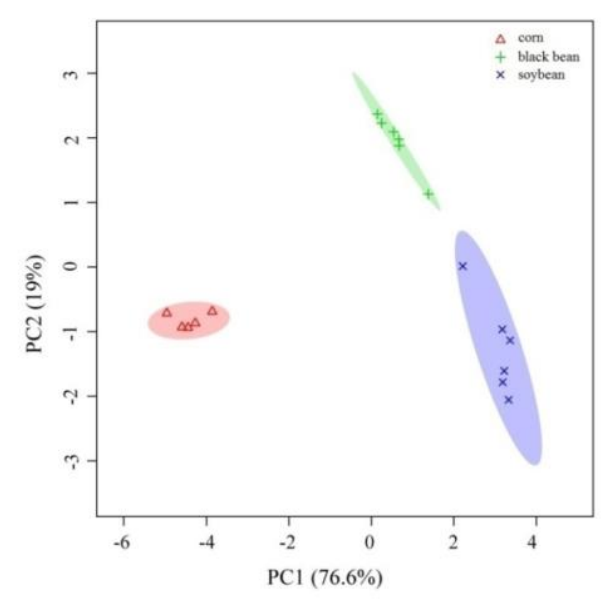

Figure 3: PCA score plot of the samples

The heat map of fatty acids in three samples was obtained with MultiExperiment View 4.9.0 (Figure 4). The relative contents of most fatty acids in soybean were higher than those in the other two. But oleic acid and linoleic acid were higher in corn and hexadecenoic acid in black bean. The unsaturated fatty acids in corn, black bean and soybean are mainly oleic acid and linoleic acid, which account for $65 \%, 57.2 \%, 50.2 \%$ of total fatty acids. The saturated fatty acids in black beans and soybean mainly include palmitic acid and stearic acid, accounting for $35.2 \%$ and $39.7 \%$ of the total fatty acids, while the saturated fatty acids in corn are mainly palmitic acids, accounting for $32.1 \%$ of the total fatty acids (Figure 5). 


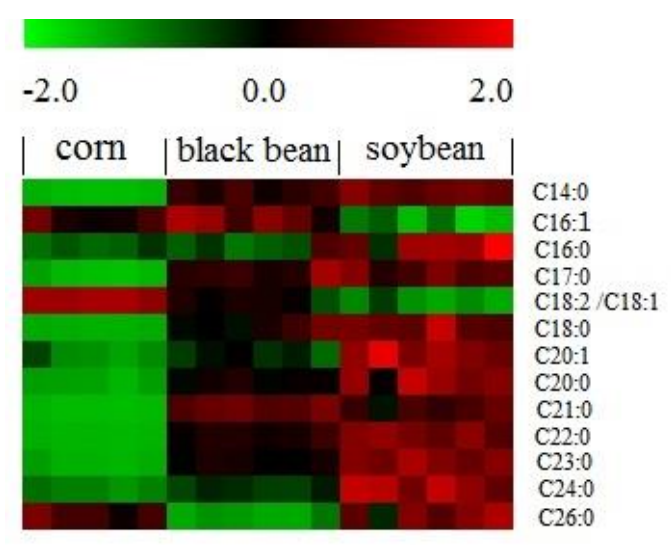

Figure 4: The heat plot of fatty acids in corn, blackbean and soybean

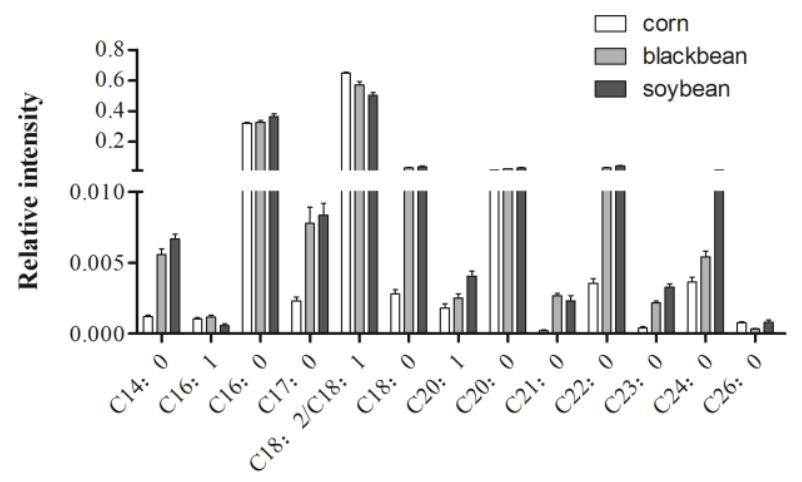

Figure 5: The relative intensity of fatty acids in corn, blackbean and soybean

\section{CONCLUSIONS}

In this study, a target analysis method based on GC-MS was established to analyze the fatty acid profile of corn, black bean and soybean. The results showed that the method was stable and reproducible and had a good linear relationship in the range of 40-140 mg sample amount. There were 14 kinds of fatty acids detected in corn, black bean and soybean. The difference of fatty acid profile between corn and black bean, soybean was greatest. The difference between black bean and soybean was relatively smaller. The relative content of most fatty acids was higher in soybean and black bean. However, unsaturated fatty acid, oleic acid and linoleic acid in corn had most content, accounting for $65 \%$ of the total amount. The method can be applied to the qualitative and quantitative analysis of fatty acids in plant seeds and provides basic data and theoretical support for the study of their nutritional value, quality improvement, variety selection and so on.

\section{ACKNOWLEDGEMENT}

This work was supported by the National Natural Science Foundation of China (grant numbers 21375046, 21605056); Project of Science and Technology Development of Jilin Province (grant number20140203013GX); Natural Science Foundation of Jilin Province (grant number20180101292JC); The financial support from the Key
Laboratory of Fine Chemicalsof Jilin Province is also acknowledged.

\section{REFERENCES}

[1] Caprioli, G., Giusti, F., Ballini, R. 2016. Lipid nutritional value of legumes: Evaluation of different extraction methods and determination of fatty acid composition. Food Chemistry, 192, 965-971.

[2] Demaison, L., Moreau, D. 2002. Dietary n-3 polyunsaturated fatty acids and coronary heart disease-related mortality: a possible mechanism of action. Cellular and Molecular Life Science, 59 (3), 463-477

[3] Heimler, D., Vignolini, P., Dini, M.G. 2005. Rapid tests to assess the antioxidant activity of phaseolus vulgaris L. Dry Beans. Journal of Agricultural and Food Chemistry, 53 (8), 3053-3056.

[4] J.R. J., F.P.C., de Castro, M.D.L. 2004. Identification and quantification of trans fatty acids in bakery products by gas chromatography-mass spectrometry after dynamic ultrasound-assisted extraction. Journal of Chromatogrphy A, 1045 (1-2), 203-210.

[5] Lunn, J., Theobald, H.E. 2006. The health effects of dietary unsaturated fatty acids. British Nutrition Foundation Nutrition Bulletin, 31 (3), 178224.

[6] Milicevic, D., Vranic, D., Masic, Z. 2014. The role of total fats, saturated unsaturated fatty acids and cholesterol conten in chicken meat as cardiovas risk factors. Lipids in Health and Disease, 13 (42), 1-12.

[7] Madhujith, T., Naczk, M., Shahidi, F. 2004. Antioxidant activity of common beans (Phaseolus vulgaris L.). Journal of Food Lipids, 11 (3), 220233.

[8] Oliva, M.L., Shannon, J.G., Sleperc, D.A. 2006. Stability of fatty acid profile in soybean genotypes with modified seed oil composition. Crop Science, 46 (5), 2069-2075.

[9] Simopoulos, A.P. 2002. Omega-3 fatty acids in inflammation and autoimmune diseases. Journal of the American College of Nutrition, 21 (6), 495-505.

[10] Wang, M.X., Zhou, D.Y., Ma, L. 2016. Analysis and evaluation of fatty acid composition in cottonseed oil. Food Science, 37 (22), 136-141.

[11] Xia, J., Wishart, D.S. 2016. Using MetaboAnalyst 3.0 for comprehensive metabolomics data analysis. Current Protocols in Bioinformatics, 55, 14.10.1-14.10.91

[12] Yank, K., Zhao, Z., Gross, R.W. 2016. Identification and quantitation of unsaturated fatty acid isomers by electrospray ionization tandem mass spectrometry: A shotgun lipidomics approach. Analytical Chemistry, 83 (11), 4243-4250.

[13] Yang, Y.Q., Zhou, Q.F., Liu, J.X. 2017. Determination of the content of main fatty acids in food legumes by gas chromatography. Journal of Food Safety and Quality, 8 (2), 574-578.

[14] Zhang, F., Bai, Y.A., Lu, H.L. 2012. Research advances in saturated fatty acid and health. China Oils and Fats, 37 (4), 29-32.

[15] Zhao, L.J. 2013. Comparison of the fatty acid in Northeast soya bean and black soya bean. Food Science and Technology, 38 (2), 155-158.

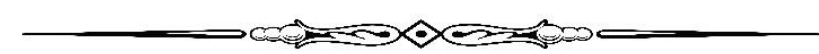

